\title{
模拟氮沉降对北京东灵山辽东栋林树木生长的影响
}

\author{
邹安龙 李修平倪晓风吉成均* \\ 北京大学城市与环境学院, 北京大学生态研究中心, 地表过程分析与模拟教育部重点实验室, 北京 100871
}

\begin{abstract}
摘 要 传统的元素限制模型认为氮是温带森林生长的限制元素, 不过该结论更多是从地上生物量以及群落水平进行阐述, 忽视了不同物种以及不同径级树木对外源氮的响应差异。辽东柇(Quercus wutaishanica)林是华北地区常见的森林类型, 该研 究以北京东灵山辽东柇林为研究对象, 通过设置 3 个氮添加水平的实验, 即对照 $\mathrm{CK}\left(0 \mathrm{~kg} \cdot \mathrm{hm}^{-2} \cdot \mathrm{a}^{-1}\right), \mathrm{N} 50\left(50 \mathrm{~kg} \cdot \mathrm{hm}^{-2} \cdot \mathrm{a}^{-1}\right)$ 和 $\mathrm{N} 100\left(100 \mathrm{~kg} \cdot \mathrm{hm}^{-2} \cdot \mathrm{a}^{-1}\right)$, 模拟氮沉降对群落和物种水平以及不同径级树木生长的影响。经过 7 年氮添加, 实验结果显示: 物种 水平上, 氮添加明显促进了优势树种辽东栎的生长; 群落水平上, 树木生长随氮浓度增加有不断上升趋势, 但统计学差异不 显著; 氮添加显著抑制了辽东柇以及群落内小径级 $(3-10 \mathrm{~cm})$ 树木生长, 中 $(10-20 \mathrm{~cm})$ 、大径级 $(>20 \mathrm{~cm})$ 树木生长随氮沉降水平 增加呈上升趋势, 但统计学差异不显著。表明氮是辽东栋以及温带森林树木生长的限制元素; 不同径级的辽东栎和群落内其 他植物对氮添加响应不一致, 氮添加抑制了小径级树木生长, 中、大径级树木生长对氮添加响应不明显。
\end{abstract}

关键词 氮沉降; 辽东柇; 树木生长; 温带森林

邹安龙, 李修平, 倪晓风, 吉成均 (2019). 模拟氮沉降对北京东灵山辽东栋林树木生长的影响. 植物生态学报, 43, 783-792. DOI: 10.17521/cjpe.2018.0232

\section{Responses of tree growth to nitrogen addition in Quercus wutaishanica forests in Mount Dongling, Beijing, China}

ZOU An-Long, LI Xiu-Ping, NI Xiao-Feng, and JI Cheng-Jun*

Institute of Ecology, College of Urban and Environmental Sciences, Laboratory for Earth Surface Processes of the Ministry of Education, Peking University, Beijing 100871, China

\section{Abstract}

Aims Temperate forest is one of the most important components of the global forests and main carbon pools. Nitrogen $(\mathrm{N})$ is considered as the limiting nutrient for the forest growth. However, the heterogeneities in plant species and stem sizes were largely ignored in previous researches on the effects of $\mathrm{N}$ addition on plant growth. Quercus wutaishanica is one of the most common and dominant tree species in the temperate forests in North China. In this study, we investigated the responses of growth of trees and forests to $\mathrm{N}$ addition in the Quercus wutaishanica forests in Mt. Dongling in Beijing.

Methods We conducted a 7-year N fertilization experiment in Quercus wutaishanica forests in Mt. Dongling, Beijing, since 2011. The $\mathrm{N}$ addition was conducted at three treatment levels, i.e., $0 \mathrm{~kg} \mathrm{hm}{ }^{-2} \cdot \mathrm{a}^{-1}, 50 \mathrm{~kg} \mathrm{hm}{ }^{-2} \cdot \mathrm{a}^{-1}$ and $100 \mathrm{~kg} \mathrm{hm}{ }^{-2} \cdot \mathrm{a}^{-1}$. Nitrogen was added at the beginning of each month from May to October each year. We used electronic vernier caliper to measure tree growth rate for each year. All trees were divided into three groups based on their diameter at breast height $(D B H)$, namely small trees $(D B H=3-10 \mathrm{~cm})$, median trees $(D B H=10-20$ $\mathrm{cm})$ and large trees $(D B H>20 \mathrm{~cm})$. Particularly, we considered growth at species level for all Quercus wutaishanica and the growth at community level for all tree species in the stands.

Important findings (1) At species level, $\mathrm{N}$ addition enhanced the growth rate of Q. wutaishanica. (2) At community level, the growth rate showed no difference among different $\mathrm{N}$ addition treatments. (3) Small trees were restrained, while median and large $Q$. wutaishanica trees were not significantly influenced, by the $\mathrm{N}$ addition.

Key words nitrogen deposition; Quercus wutaishanica; tree growth; temperate forest

Zou AL, Li XP, Ni XF, Ji CJ (2019). Responses of tree growth to nitrogen addition in Quercus wutaishanica forests in Mount Dongling, Beijing, China. Chinese Journal of Plant Ecology, 43, 783-792. DOI: 10.17521/cjpe.2018.0232

收稿日期Received: 2018-09-18 接受日期Accepted: 2019-02-28

基金项目：国家自然科学基金(31770431)和国家科技基础性工作专项(2011FY110300)。Supported by the National Natural Science Foundation of China (31770431), and the National Basic Work of Science and Technology of China (2011FY110300).

* 通信作者Corresponding author (jicj@pku.edu.cn) 
元素限制模型是预测全球植被生产力变化的重 要工具, 该模型认为氮 $(\mathrm{N})$ 和磷 $(\mathrm{P})$ 是限制植物生长 的重要元素, 外源氮和磷添加能促进植物生长, 提 高植物生产力(Aber et al., 1989; Jung et al., 2011; Du et al., 2016)。传统观点认为, 在热带和亚热带等低 纬度地区，树木生长受磷限制，而暖温带和寒温带 等中高纬度区域, 树木生长主要受氮限制(Magnani et al., 2007; Vitousek et al., 2010)。在模拟施肥实验 中, 前人主要通过监测树木生物量的变化来探究限 制元素对树木生长的影响(Gundersen et al., 1998; Wright \& Rasmussen, 1998; Jefts et al., 2004; Magill et al., 2004)。由于大径级树木的地上生物量对群落 生物量的贡献度要大于小径级树木, 以前的研究往 往忽视了限制元素对小径级树木生长的影响(Li et al., 2018)。其次, 传统限制模型主要从群落水平研 究树木生长对限制元素的响应情况, 而忽略了不同 物种的响应(Aber et al., 1995; Matson et al., 2002; Solberg et al., 2009; de Vries et al., 2014)。Li等(2018) 在暖温带和寒温带地区进行的施肥实验表明, 元素 的限制作用对小径级树木生长表现明显, 对大径级 树木以及整体群落植物生长无显著影响。Magill等 (2004)对温带森林的研究指出, 氮添加促进了硬木 林植物的生长, 抑制了红松林植物的生长。因此, 需 要分径级和分树种探究限制元素对树木生长的影响, 以期对传统的元素限制模型进行补充和完善。

不同径级的树木对营养元素的响应机理存在差 异。一方面, 小树处于群落低层, 对光的竞争能力低 于大树, 因此摄入的营养元素主要用于促进生长或 者提高叶片元素含量从而提高光合效率, 以维持自 身的生长; 而大树由于生长时间较长以及资源配置 需要, 对元素的需求更多用于满足繁殖和维持呼吸 代谢, 在生长方面的投资相对较小(Burslem et al., 1995)。另一方面, 小树存储的营养元素小于大树, 对养分元素含量的变化更敏感(Deng et al., 2016)。 综合两方面原因, 营养元素对小树的影响可能要大 于大树。不同物种对限制元素的响应也有所区别。 以氮为例, 一方面, 不同植物对氮的利用效率存在 差异, 对氮的转化速率快慢直接影响植物在群落中 的生存状况(Gilliam, 2006; 鲁显楷等, 2008)。另一方 面, 氮添加会造成土壤酸化, 打破土壤中的元素平 衡(Nadelhoffer et al., 1999), 致使对土壤环境变化敏 感的植物竞争力降低, 生长受抑制甚至消失。
相关研究表明, 温带森林植物生长对氮添加的 响应可能较慢, 一般需要 5 年以上的时间(Gundersen et al., 1998; Kjonaas et al., 1998)。不同氮沉降速率对 树木生长的影响也不尽相同。有研究指出, 氮添加 水平高于 $25 \mathrm{~kg} \cdot \mathrm{hm}^{-2} \cdot \mathrm{a}^{-1}$ 时, 植物的生长会受影响 (Stevens, 2004; Reidsma et al., 2006), 也有研究认为, 当氮添加水平超过 $10 \mathrm{~kg} \cdot \mathrm{hm}^{-2} \cdot \mathrm{a}^{-1}$ 时, 会抑制树木的 生长(Clark \& Tilman, 2008)。然而, 大多数施肥对树 木生长影响实验的持续时间一般比较短, 而且元素 添加水平较少。因此, 在温带森林开展长时间并且 多水平的元素添加实验, 才能更好地预测限制元素 对树木生长的影响。

辽东栋(Quercus wutaishanica) 是华北地区常见 的落叶阔叶优势树种, 广泛分布在黄河流域以及辽 东半岛等地, 以其为主要种或优势种组成的群落属 于较为稳定的森林植被类型。辽东栋的外貌、结构 以及动态变化对于暖温带植被类型具有很好的指示 作用(王巍等, 2000)。本研究以北京东灵山辽东栋林 为研究对象, 通过连续7年氮添加梯度实验, 试图回 答以下两个问题: 1) 在物种水平, 氮添加对优势种 辽东栎生长有何影响? 2)在群落水平, 氮添加对不 同径级树木生长有何影响?

\section{1 材料和方法}

\section{1 样地概况}

研究地点位于北京东灵山森林生态系统定位研 究站 $\left(39.97^{\circ} \mathrm{N}, 115.43^{\circ} \mathrm{E}\right)$, 海拔 $1250 \mathrm{~m}$ 。该站点为 中国森林生态系统养分添加实验网络(NEECF)平台 站点之一, 为长期氮添加实验标准区域(Du et al., 2013)。该地区属于温带半湿润季风气候, 夏季高温 多雨, 冬季寒冷干燥。年降水量 $(570.3 \pm 112.2) \mathrm{mm}$, 大部分集中在6-8月份; 年平均气温 $(4.9 \pm 0.6){ }^{\circ} \mathrm{C}$, 最热月 $\left(7\right.$ 月)平均气温 $(18.7 \pm 1.0){ }^{\circ} \mathrm{C}$, 最冷月 $(1$ 月) 平均气温 $(-10.4 \pm 1.5){ }^{\circ} \mathrm{C}$ 。棕壤土, 土层厚度为 $90-$ $120 \mathrm{~cm}, \mathrm{pH}$ 值为6.3-6.9 (苏宏新和李广起, 2012)。

实验样地位于山腰西南向阳坡, 植被保存完好, 物种丰富, 层次分明。乔木层植物主要包括辽东栎、 大叶白蜡 (Fraxinus rhynchophylla)、黑桦 (Betula dahurica) 和大果榆(Ulmus macrocarpa) 等, 其中辽 东栋为优势物种; 乔木亚层主要由色木槭(Acer mono)构成; 林下灌木常见物种包括六道木(Abelia biflora)、土庄绣线菊(Spiraea pubescens)、大花舅疏 
(Deutzia grandiflora)、小花舅疏(D. parviflora)、胡 枝子(Lespedeza bicolor)等; 草本植物繁茂, 主要包 括野青茅(Deyeuxia arundinacea)、蒙古蒿(Artemisia mongolica)、银背风毛菊(Saussurea nivea)等。

\section{2 实验设计}

氮添加实验开始于 2011 年10月中旬, 共设置对 照CK $\left(0 \mathrm{~kg} \cdot \mathrm{hm}^{-2} \cdot \mathrm{a}^{-1}\right) 、 \mathrm{~N} 50\left(50 \mathrm{~kg} \cdot \mathrm{hm}^{-2} \cdot \mathrm{a}^{-1}\right)$ 和N100 $\left(100 \mathrm{~kg} \cdot \mathrm{hm}^{-2} \cdot \mathrm{a}^{-1}\right) 3$ 种氮添加水平处理, 每种处理 3 个重复。样方大小为 $20 \mathrm{~m} \times 20 \mathrm{~m}$, 样方之间设置 $10 \mathrm{~m}$ 的过渡带。实验采用完全随机实验, 选取立地 条件接近的样地以最小化空间异质性。从2012年开 始, 每月初, 将相应质量的尿素 $\left(\mathrm{CO}\left(\mathrm{NH}_{2}\right)_{2}\right)$ 溶于 30 L水中, 彻底溶解后用喷雾器均匀喷酒于氮添加 样方，对照样方仅喷酒相同质量的水，施肥时间为 每年的5-10月。

\section{3 实验处理}

2011年 10 月, 用精度为 $0.1 \mathrm{~cm}$ 的软尺对样方所 有植物测量初始胸径( $D B H$, 树高 $1.3 \mathrm{~m}$ 处), 并将样 方内所有 $D B H \geqslant 3 \mathrm{~cm}$ 的树进行编号, 挂牌, 安装 铝制自动测树器(Alvarez-Clare et al., 2013)。待测树 器稳定后, 从2011年至2017年, 每年的11月初用精 度为 $0.01 \mathrm{~mm}$ 的电子游标卡尺测量测树器窗口的变 化。采用年际间窗口大小的差值计算树木的胸径增 长量(公式1):

$$
D B H_{i}=D B H_{i-1}+\frac{X_{i}-X_{i-1}}{\pi}
$$

其中, $D B H_{i}$ 表示当年胸径, $D B H_{i-1}$ 表示上一年胸径, $X_{i}$ 表示该年窗口值, $X_{i-1}$ 表示上一年窗口值, $\pi$ 表示圆 周率。

根据下面公式计算样方植物的胸高断面积 $(B A)$ 。

$$
B A=\left(\frac{D B H}{2}\right)^{2} \times \pi
$$

根据下面公式计算植物相对增长速率 $(R G R)$ 。

$$
R G R=\frac{B A_{i}-B A_{i-1}}{B A_{i-1}}
$$

其中, $B A_{i}$ 表示该年胸高断面积, $B A_{i-1}$ 表示上一年植 物胸高断面积。

七年总的植物相对增长速率 $\left(R G R_{7}\right)$ 通过以下公 式求得:

$$
R G R_{7}=\frac{T B A(2017)-T B A(2011)}{T B A(2011)} \times 100 \%
$$

其中, TBA(2017)表示群落植物2017年胸高断面积总 和, TBA(2011)表示群落植物2011年胸高断面积总和。

\section{4 样品采集与测定}

在2015年8月底(距离上次氮添加时间间隔近一 个月), 在每个样方随机取 5 个点, 用内径为 $5 \mathrm{~cm}$ 的 土钻采集0-10 cm表层土壤，带回实验室风干，在剔 除石块和植物残根之后，粉碎并过 $2 \mathrm{~mm}$ 篮，充分混 匀。称取 $15 \mathrm{~g}$ 风干土壤, 用 $50 \mathrm{~mL} \mathrm{KCl}\left(1 \mathrm{~mol} \cdot \mathrm{L}^{-1}\right)$ 溶 液浸提, 用酸度计 (pH S-3B, 上海精科, 上海)测定 $\mathrm{pH}$ 值。另取 $50 \mathrm{~g}$ 自然风干的土壤样品, 研磨充分后, 使用元素分析仪(Elementar Vario EL III, Hanau, Germany)测定土壤全氮(TN)和全碳(TC)含量, 用钼 锑抗比色法测定土壤全磷(TP) 含量(王乔姝怡等, 2016)。

在2017年8月初(距离上次氮添加时间间隔一个 月左右), 在每个样方内分别随机挑选5株辽东柇植 株。每株植株采集向阳、林冠层 $1 / 3$ 处的 20 片长势健 康的叶片, 带回实验室至 $65{ }^{\circ} \mathrm{C}$ 烘箱烘干 $48 \mathrm{~h}$ 至恒质 量, 用元素分析仪测定叶片全氮(TN)含量和全碳 (TC)含量, 用钼锑抗比色法测定叶片全磷(TP)含量。

\section{5 数据分析}

依据树木胸径大小将实验样地植物分为: 小 $(3-10 \mathrm{~cm}) 、$ 中 $(10-20 \mathrm{~cm})$ 和大 $(>20 \mathrm{~cm}) 3$ 个径级, 剔 除物种以及胸径本底值的影响, 研究氮添加对树木 生长的影响(Tian et al., 2017)。其中, 利用单因素方 差分析研究不同氮添加水平对土壤理化性质, 物种 水平和群落水平以及不同径级树木相对增长速率等 指标的差异性。 $p<0.05$ 时, 利用最小显著性差异法 $(L S D)$ 对不同径级树木的相对增长速率进行多重比 较。利用线性回归方程检验土壤元素与树木相对增 长速率以及叶片元素含量的相关性。用SigmaPlot 12.0 完成制图, 用SPSS 24.0进行数据分析。

\section{2 结果}

\section{1 氮添加对土壤属性的影响}

随着氮添加浓度升高, 表层土壤中TC以及 $\mathrm{TN}$ 含量有不断上升的趋势, N100处理下 TC 和TN含量 均值要高于 $\mathrm{CK}$ 处理(图1 A、1B)。氮添加微弱提高了 高氮处理(N100)土壤的TP含量 $(p=0.08)$ (图1C)。土 壤 $\mathrm{pH}$ 值随氮添加水平升高有下降趋势, 但统计学差 异不显著, 3 种处理的 $\mathrm{pH}$ 值分别为6.53、6.13和 6.08 (图1D)。 


\section{2 氮添加对辽东栋叶片元素含量变化的影响}

如图2所示，氮添加对辽东栎叶片 $\mathrm{TC}$ 和 TP含量 均无显著影响，但显著提高了叶片 $\mathrm{TN}$ 含量 $(p<$ $0.05), \mathrm{CK} 、 \mathrm{~N} 50$ 和 $\mathrm{N} 100$ 三种处理叶片 $\mathrm{TN}$ 含量分别为 $2.37 、 2.83$ 和 $2.72 \mathrm{mg} \cdot \mathrm{g}^{-1}$ 。

\section{3 氮添加对群落植物以及优势树种辽东栋生长} 的影响

从物种水平上看, 不同处理间辽东栎相对增长 速率 $(R G R)$ 差异显著 $(p<0.05)$, 三种处理的 $R G R$ 分
别为 $17.52 \% 、 19.76 \%$ 和 $24.34 \%$, 说明氮处理促进了 辽东柇树木的生长(图3A)。从群落水平上看，植物 $R G R$ 随氮添加水平升高有不断上升趋势, 但在统计 学上差异不显著 $(p>0.05), \mathrm{CK} 、 \mathrm{~N} 50$ 和 $\mathrm{N} 100$ 三种氮 添加处理的 $R G R$ 分别为 $17.11 \% 、 17.13 \%$ 和 $23.02 \%$ (图3B)。

进一步研究显示, 表层土壤元素中, TN含量与 群落植物以及辽东栎的 $R G R$ 之间存在显著的线性关 系(图4C、4D) $(p=0.025,0.007)$ 。但是土壤元素和辽
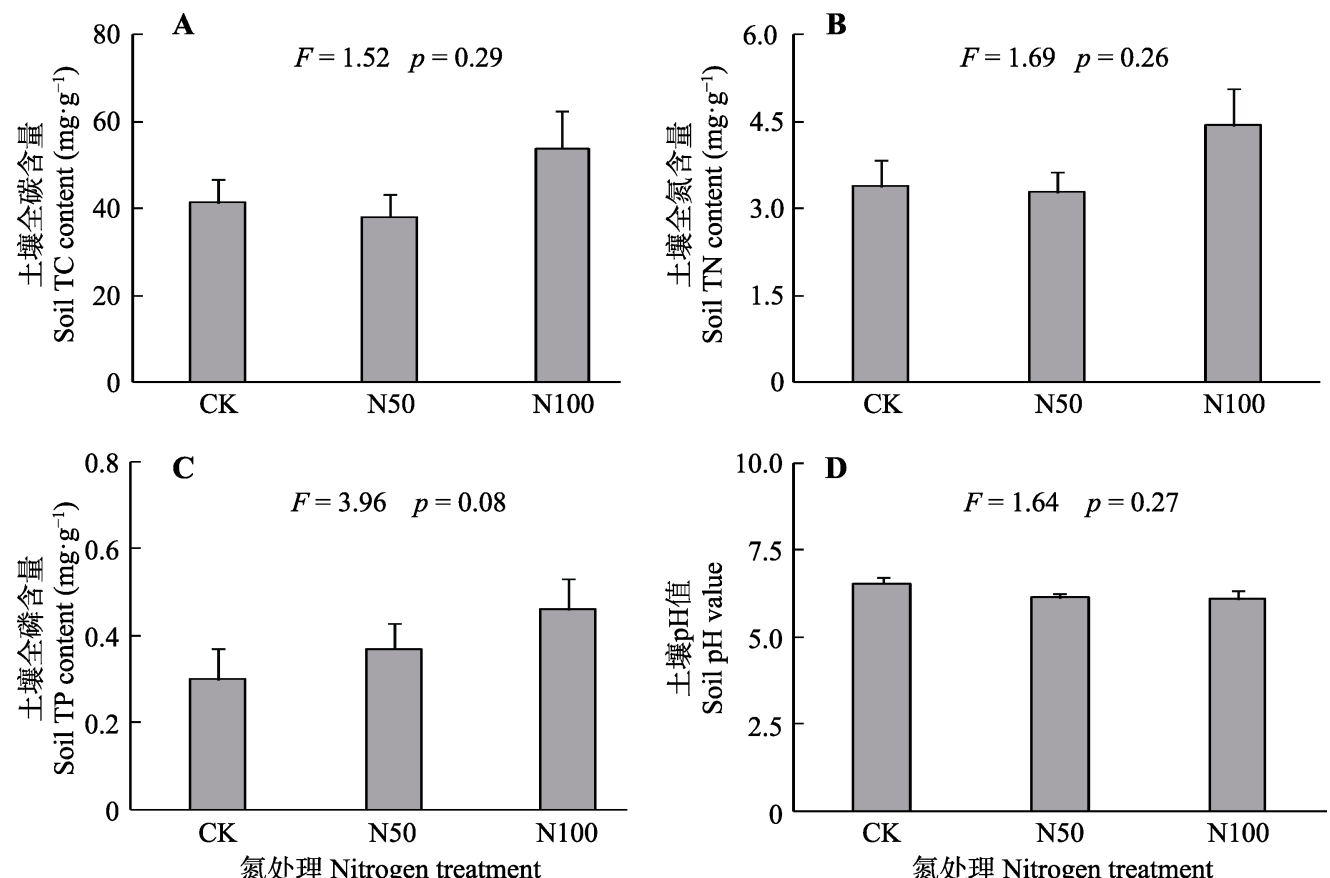

图1 氮添加对北京东灵山辽东栋林土壤全碳(TC)、全氮 $(\mathrm{TN})$, 全磷(TP)含量以及 $\mathrm{pH}$ 值的影响(平均值+标准误差, $n=3)$ 。不 同小写字母表示处理间差异显著 $(p<0.05)$ 。CK、N50、N100分别表示氮添加量0、50、100 kg $\cdot \mathrm{hm}^{-2} \cdot \mathrm{a}^{-1}$ 。

Fig. 1 Influence of nitrogen addition treatments on soil total carbon (TC), total nitrogen (TN), total phosphorus (TP) content and pH value in Quercus wutaishanica forests in Mt. Dongling, Beijing (mean $+S E, n=3$ ). Different lowercase letters indicate significant difference among treatments $(p<0.05)$. CK, N50, N100 denote nitrogen addition $0,50,100 \mathrm{~kg} \cdot \mathrm{hm}^{-2} \cdot \mathrm{a}^{-1}$, respectively.
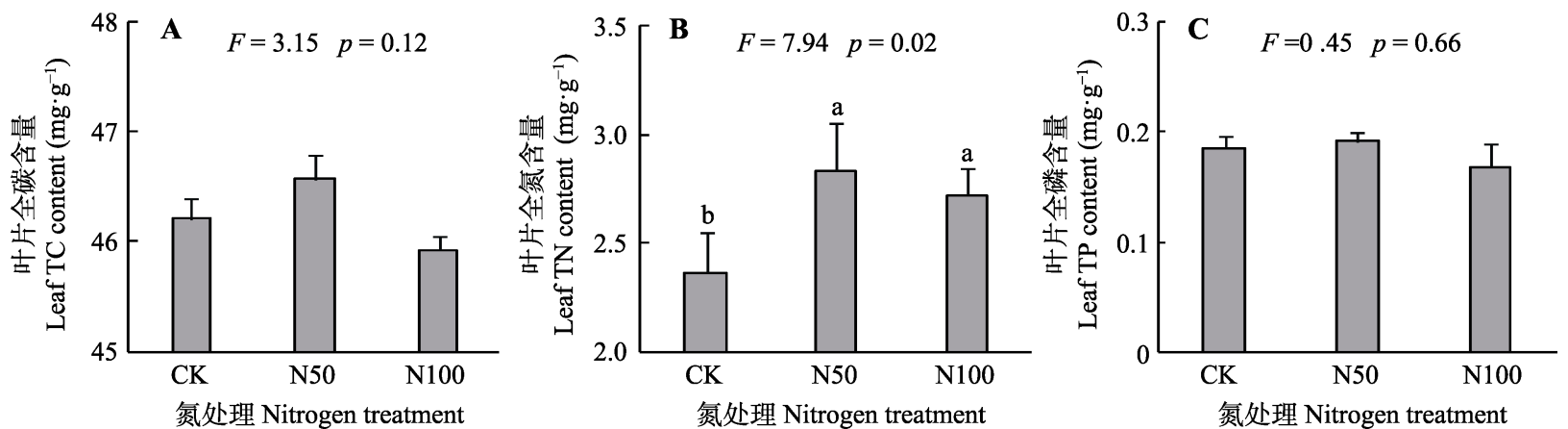

图2 氮添加对北京东灵山辽东栎叶片全碳(TC)、全氮 $(\mathrm{TN})$ 、全磷(TP)含量的影响(平均值+标准误差, $n=3)$ 。不同小写字母 表示处理间差异显著 $(p<0.05)$ 。CK、N50、N100分别表示氮添加量 $0 、 50 、 100 \mathrm{~kg} \cdot \mathrm{hm}^{-2} \cdot \mathrm{a}^{-1}$ 。

Fig. 2 Influence of nitrogen additions on leaf total carbon (TC), total nitrogen (TN), total phosphorus (TP) content of Quercus wutaishanica in Mt. Dongling, Beijing (mean $+S E, n=3$ ). Different lowercase letters indicate significant difference among treatments $(p<0.05)$. CK, N50, N100 denote nitrogen addition $0,50,100 \mathrm{~kg} \cdot \mathrm{hm}^{-2} \cdot \mathrm{a}^{-1}$, respectively. 

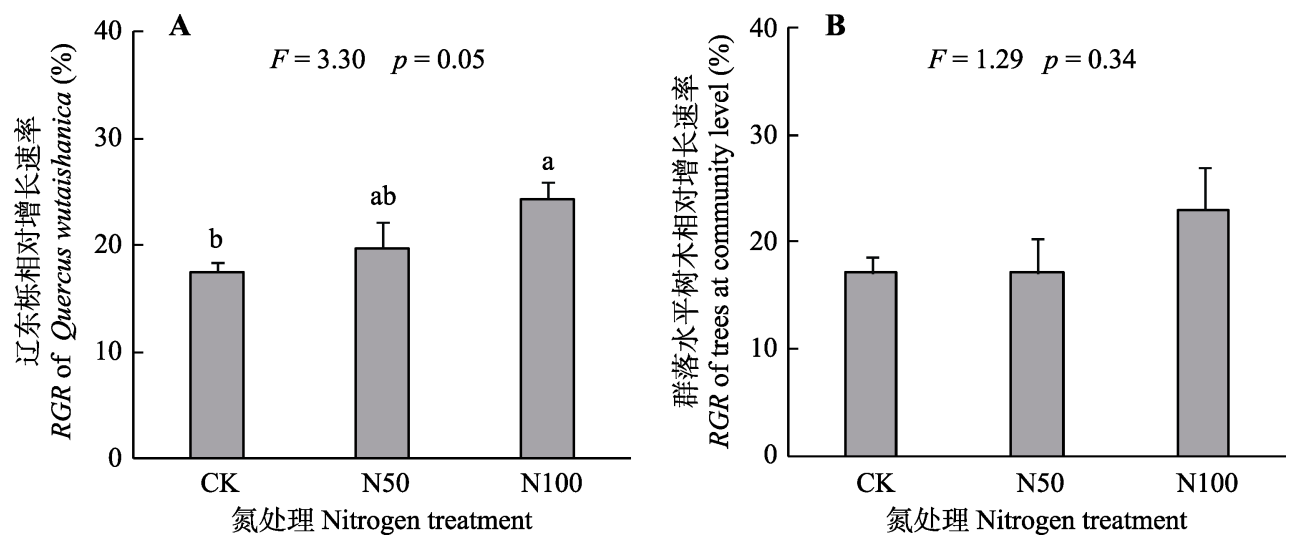

图3 氮添加对北京东灵山辽东栎以及群落水平树木相对增长速率 $(R G R)$ 变化的影响(平均值+标准误差, $n=3)$ 。不同小写字母 表示处理间差异显著 $(p<0.05)$ 。CK、N50、N100分别表示氮添加量 $0 、 50 、 100 \mathrm{~kg} \cdot \mathrm{hm}^{-2} \cdot \mathrm{a}^{-1}$ 。

Fig. 3 Influence of nitrogen addition treatments on the relative growth rate (RGR) of basal area of Quercus wutaishanica and trees at community level in Mt. Dongling, Beijing (mean $+S E, n=3$ ). Different lowercase letters indicate significant difference among treatments $(p<0.05)$. CK, N50, N100 denote nitrogen addition $0,50,100 \mathrm{~kg} \cdot \mathrm{hm}^{-2} \cdot \mathrm{a}^{-1}$, respectively.
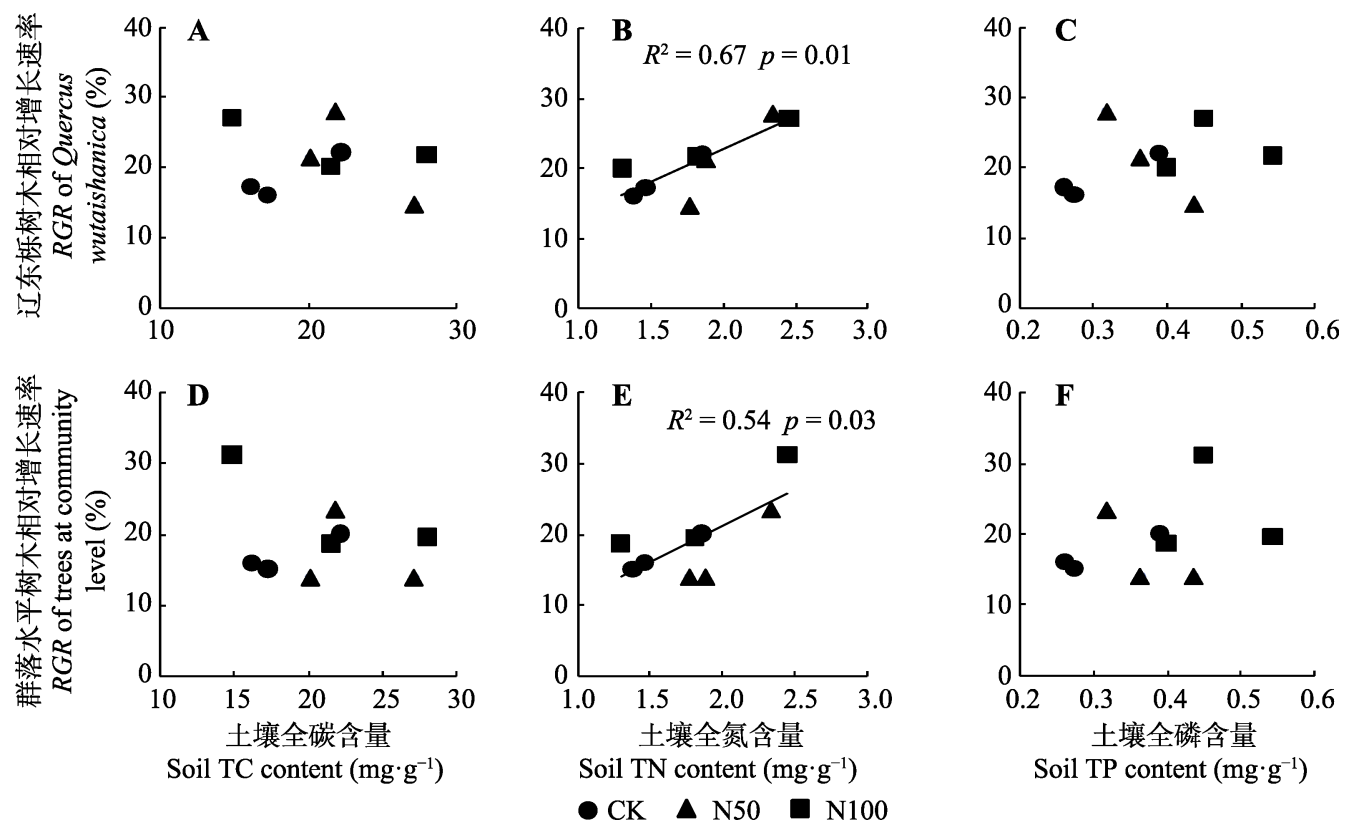

图4 北京东灵山土壤全碳( $\mathrm{TC}) 、$ 全氮 $(\mathrm{TN})$ 和全磷 $(\mathrm{TP})$ 含量与辽东栎以及群落水平树木相对增长速率 $(R G R)$ 的关系。每一个 点表示该样方的平均值。CK、N50、N100分别表示氮添加量0、50、100 $\mathrm{kg} \cdot \mathrm{hm}{ }^{-2} \cdot \mathrm{a}^{-1}$ 。

Fig. 4 Relationships of relative growth rate $(R G R)$ of Quercus wutaishanica and trees at community level with soil total carbon (TC), total nitrogen (TN) and total phosphorus (TP) content in Mt. Dongling, Beijing. Each dot indicates the mean in each plot. CK, $\mathrm{N} 50, \mathrm{~N} 100$ denote nitrogen addition $0,50,100 \mathrm{~kg} \cdot \mathrm{hm}^{-2} \cdot \mathrm{a}^{-1}$, respectively.

东柇叶片元素之间无显著线性关系 $(p>0.05)$ (图5)。

\section{4 氮添加对不同径级植物生长的影响}

在物种水平和群落水平, 不同径级植物的 $R G R$ 对氮添加的响应不同，但同一径级植物的 $R G R$ 对氮 添加的响应呈现出一致的规律。其中, 氮添加显著 抑制了小径级 (3-10 cm) 树木的生长 $(p<0.05)$, 而中 $(10-20 \mathrm{~cm})$ 、大径级 $(>20 \mathrm{~cm})$ 树木的 $R G R$ 随着氮浓度 升高有不断上升的趋势, 但统计学差异不显著 $(p>$ 0.05)(图6A、6B)。

在物种水平和群落水平, 中、大径级树木 $R G R$
随表层土壤 $\mathrm{TN}$ 含量升高而显著增大 $(p<0.05)$, 小 径级树木 $R G R$ 与土壤 $\mathrm{TN}$ 含量无显著线性关系 $(p>$ 0.05)(图7)。

\section{3 分析和讨论}

\section{1 优势树种辽东栋以及群落植物对氮添加的响应}

资源的可利用性影响着生态系统的存续和发展, 其中营养元素是所有资源中最重要的一项。当一种 或者几种营养元素含量不足或偏低时，植物的生长 会受到抑制。通过外源元素添加能够提高土壤中该 

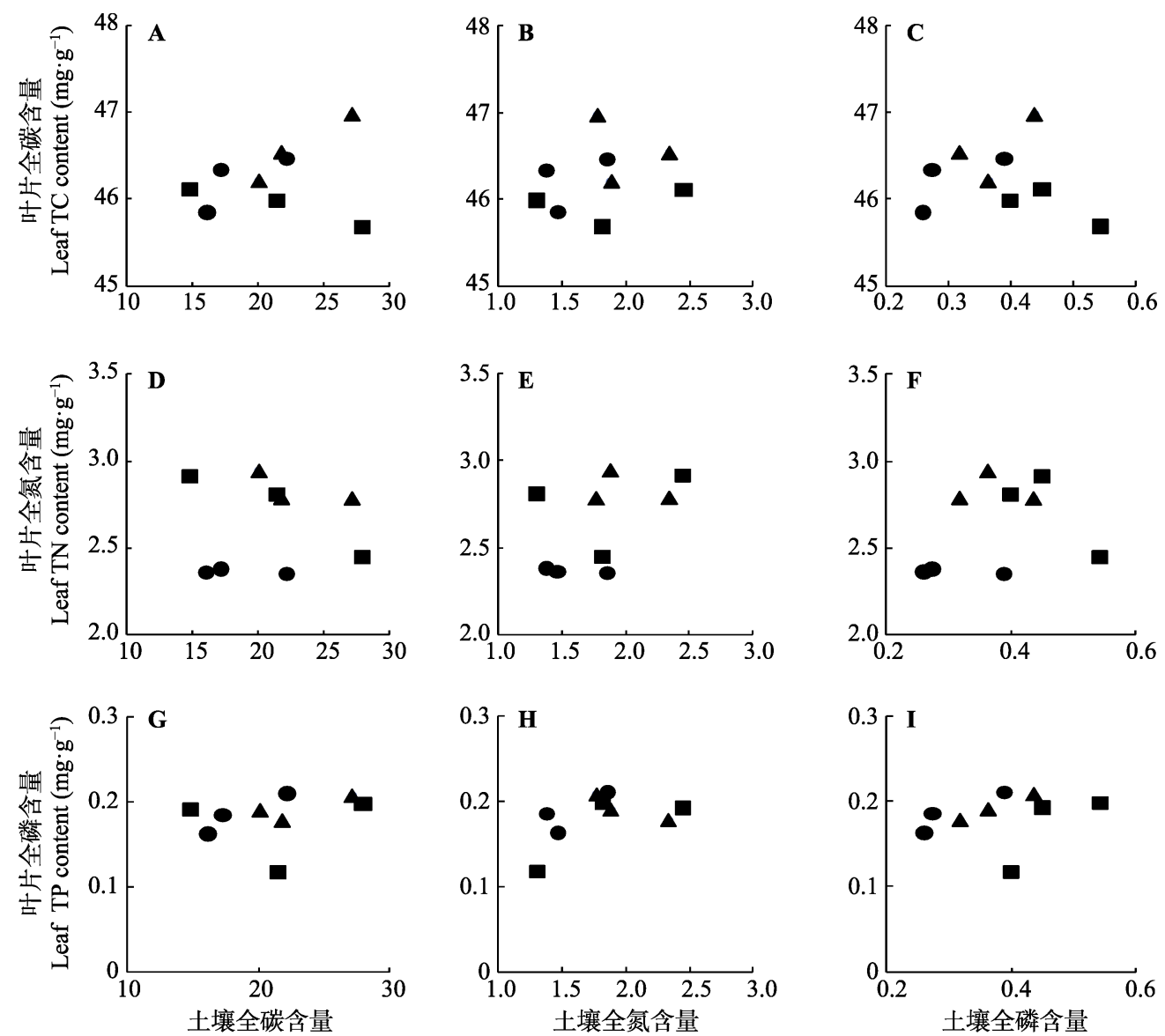

Soil TN content $\left(\mathrm{mg} \cdot \mathrm{g}^{-1}\right)$

$\bullet \mathrm{CK} \triangle \mathrm{N} 50 \quad \mathrm{~N} 100$

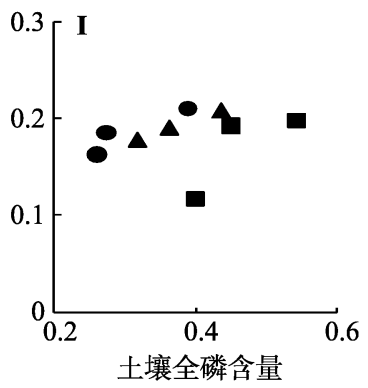

Soil TP content $\left(\mathrm{mg} \cdot \mathrm{g}^{-1}\right)$

图5 北京东灵山土壤全碳( $\mathrm{TC})$ 、全氮(TN)和全磷(TP)含量与辽东栎叶片全碳、全氮和全磷含量的关系。每一个点表示该样 方的平均值。CK、N50、N100分别表示氮添加量 $0 、 50 、 100 \mathrm{~kg} \cdot \mathrm{hm}^{-2} \cdot \mathrm{a}^{-1}$ 。

Fig. 5 Relationships of Quercus wutaishanica leaf total carbon (TC), total nitrogen (TN) and total phosphorus (TP) content with soil total carbon, total nitrogen and total phosphorus content in Mt. Dongling, Beijing. Each dot indicates the mean in each plot. CK, N50, $\mathrm{N} 100$ denote nitrogen addition $0,50,100 \mathrm{~kg} \cdot \mathrm{hm}^{-2} \cdot \mathrm{a}^{-1}$, respectively.
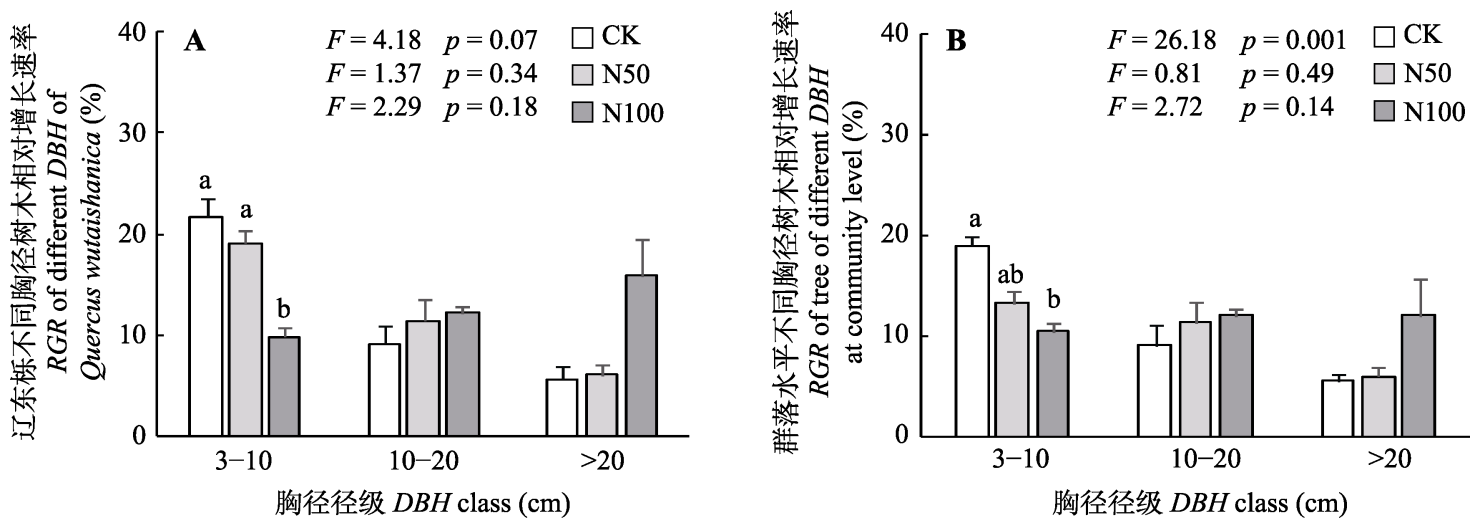

图6 氮添加对北京东灵山辽东栎以及群落水平不同径级 $(D B H)$ 树木相对增长速率 $(R G R)$ 的影响(平均值+标准误差, $n=3)$ 。不 同小写字母表示处理间差异显著 $(p<0.05)$ 。3-10、10-20、>20分别表示植物胸径 $(D B H)$ 范围为3-10、10-20、>20 cm。CK、 $\mathrm{N} 50 、 \mathrm{~N} 100$ 分别表示氮添加量0、50、100 $\mathrm{kg} \cdot \mathrm{hm}^{-2} \cdot \mathrm{a}^{-1}$ 。

Fig. 6 Influence of nitrogen addition treatments on the relative growth rate $(R G R)$ of different diameter at breast height $(D B H)$ classes of Quercus wutaishanica and trees at community level in Mt. Dongling, Beijing (mean $+S E, n=3$ ). Different lowercase letters indicate significant difference among treatments $(p<0.05)$. 3-10, 10-20, $>20$ denote trees diameter at breast height $(D B H)$ range are $3-10,10-20,>20 \mathrm{~cm}$. CK, N50, N100 denote nitrogen addition $0,50,100 \mathrm{~kg} \cdot \mathrm{hm}^{-2} \cdot \mathrm{a}^{-1}$, respectively. 

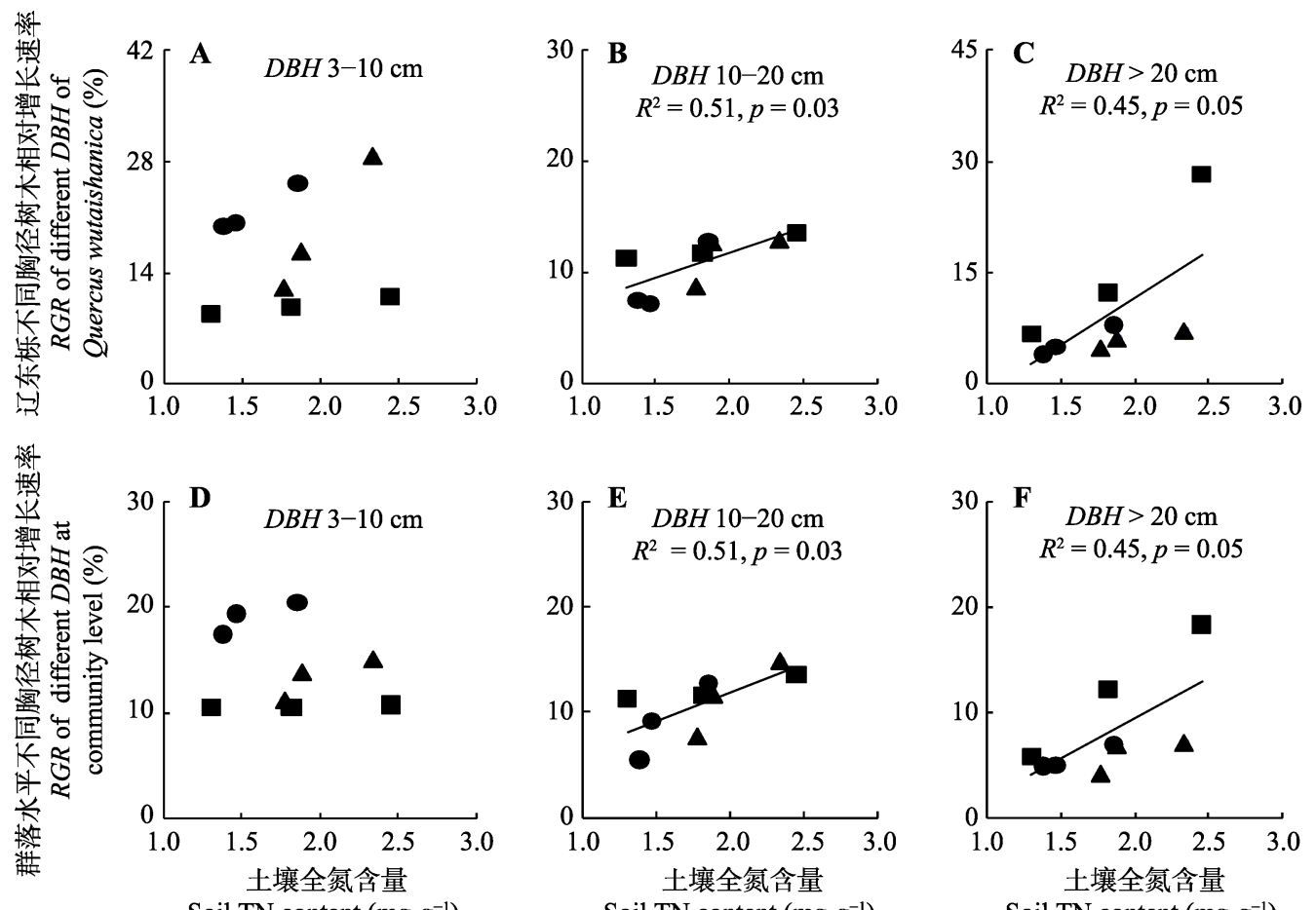

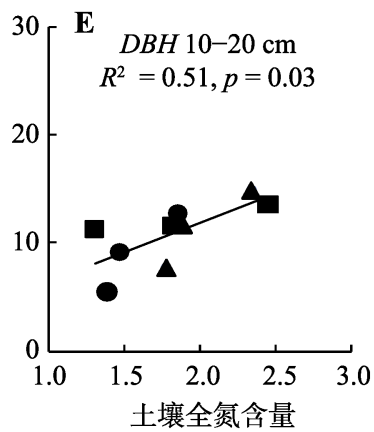

Soil TN content $\left(\mathrm{mg} \cdot \mathrm{g}^{-1}\right)$

- CK $\triangle$ N50 $\quad \mathrm{N} 100$

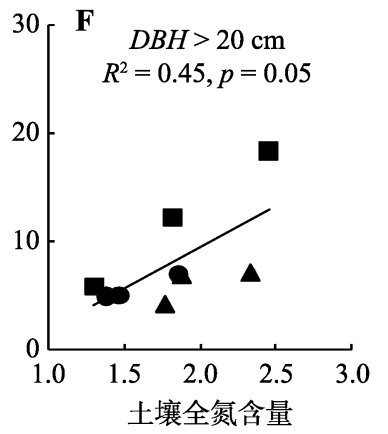

Soil TN content $\left(\mathrm{mg} \cdot \mathrm{g}^{-1}\right)$

图7 北京东灵山土壤全氮 $(\mathrm{TN})$ 含量与辽东栋以及群落水平不同径级 $(D B H)$ 树木相对增长速率 $(R G R)$ 的关系。每一个点表示该 样方的平均值。CK、N50、N100分别表示氮添加量0、50、100 $\mathrm{kg} \cdot \mathrm{hm}^{-2} \cdot \mathrm{a}^{-1}$ 。

Fig. 7 Relationships of relative growth rate $(R G R)$ of different diameter at breast height (DBH) classes of Quercus wutaishanica in Mt. Dongling, Beijing and trees at community level with soil nitrogen (TN). CK, N50, N100 denote nitrogen addition 0, 50, 100 $\mathrm{kg} \cdot \mathrm{hm}^{-2} \cdot \mathrm{a}^{-1}$, respectively.

营养元素含量, 进而减缓营养元素对生态系统的限 制作用(Koerselman \& Meuleman, 1996)。当植物或 生态系统对外源元素添加呈积极响应，比如促进植 物生长或提高地上生物量, 就可认定该元素为植物 或生态系统的限制元素(Vitousek \& Howarth, 1991; Alvarzclare et al., 2013)。我们的研究(图3)发现, 优 势树种辽东栋以及群落水平植物的 $R G R$ 整体上对氮 添加呈积极响应(Högberg et al., 2006; BassiriRad et $a l .$, 2015), 说明外源氮添加能够促进优势树种以及 辽东栋群落的生长。由此推断, 氮可能是辽东栋以 及暖温带森林生态系统的限制元素, 本研究结果与 传统的元素限制理论一致(Magnani et al., 2007; Vitousek et al., 2010)。

群落水平植物的 $R G R$ 随氮添加呈不断上升趋势, 但差异不显著的原因可能来自诸多方面。其一, 前 人的研究表明, 氮添加会导致土壤酸化, 降低土壤 $\mathrm{pH}$ 值, 从而影响某些对土壤酸性变化敏感的植物的 生长(Bergkvist \& Folkeson, 1992; 孙本华等, 2006)。 我们的结果显示, 氮添加有降低表层土壤 $\mathrm{pH}$ 值的趋 势, 由于群落水平物种较多, 因此有可能抑制了某
些树种的生长(图1D), 而辽东栋属于群落的优势种, 所占比重较大, 且其生长对氮添加呈积极响应, 可 能导致了整体群落水平树木生长表现出积极响应。 其二, 由于温带地区较低的年降水量和较短的植物 生长期，限制了植物对养分的吸收利用，导致短期 的氮添加对植物生长的影响不显著。Kjonaas等 (1998)在瑞典针叶林开展的氮沉降实验显示, 持续5 年的氮添加并未改变树木的生长速度。尽管我们的 实验时间有7年，但相对而言还是比较短的。而且有 研究表明, 样地的空间异质性以及氮的形式会影响 施肥效率(Wright et al., 2011)。由于本实验样地地势 比较陡, 且实验施肥原料是尿素, 在植物利用上仍 属于铵态氮, 挥发性较强, 减弱了施肥效果。因此关 于氮添加对植物生长的影响还需要持续观测研究。

将辽东栋和群落植物分为小 $(3-10 \mathrm{~cm})$ 、中 $(10-20 \mathrm{~cm})$ 和大 $(>20 \mathrm{~cm}) 3$ 个径级后, 发现不同径级 辽东栋和群落植物对氮添加响应趋势不一致(图6): 氮添加明显抑制了小径级树木的生长, 氮添加对中 径级和大径级树木的生长有促进作用, 但响应不显 著(Tian et al., 2017; Li et al., 2018)。通常认为, 外源 
元素的输入若超过了植物本身对该元素的同化作用, 不仅会影响植物对该元素的吸收, 同时也会破坏植 物体内其他营养元素化学计量耦合关系的平衡, 从 而抑制植物生长(Harpole et al., 2011)。小径级树木 对环境的适应能力以及对外源元素的同化作用和维 持化学计量耦合关系的稳定性要弱于中、大径级树 木, 而且元素吸收和转化能力比中、大径级树木也 更弱。因此, 对元素变化更敏感的小径级树木, 很有 可能因为外源元素的输入打乱了化学计量耦合关系 的平衡, 从而表现为抑制生长。

\section{2 植物对外源元素的利用策略}

光照是限制森林内部植物生长的主要因子, 为 了获取更多的光资源, 植物需要吸收更多的营养元 素, 用于加快树木生长速率或(和)提高叶片养分含 量以提高光合效率, 从而提高对光资源的竞争力 (McConnaughay \& Coleman, 1999; Uriarte et al., 2005)。从物种水平以及群落水平上看, 土壤 $\mathrm{TN}$ 含量 和辽东栋以及群落植物 $R G R$ 之间存在显著的线性关 系(图4), 说明辽东栋树种以及群落植物对氮需求旺 盛, 可以将土壤中的氮转化成生长所必需的营养成 分, 也进一步说明氮可能是该地区的限制元素。此 外, 辽东栎叶片元素含量与土壤元素含量之间的关 系不明显(图5), 说明外源氮可能更多被用于辽东栋 树木的生长, 而不是储存在叶片中。这可能是因为 辽东栎属于优势种, 大径级个体一般处于群落的上 层, 获取光照的能力普遍高于其他植物, 对养分用 于生长的需求大于用于光照的需求。

不同径级植物对养分的利用策略不同。一般认 为, 小径级树木长得相对矮小, 在和中、大径级树木 竞争光资源中处于弱势地位。为了提高竞争力, 小 径级树木吸收的营养元素, 既要用于促进植物生长, 也要用于增加叶片养分含量, 从而提高光合效率

(Burslem et al., 1995; Li et al., 2018)。我们的研究结 果显示, 土壤 $\mathrm{TN}$ 含量与辽东栋树种以及群落水平 的中、大径级树木 $R G R$ 有很好的线性关系, 而与小径 级辽东柇树种以及群落水平小径级树木的 $R G R$ 关系 不明显(图7)。这可能说明中、大径级树木将氮更多 用于木材纵向生长, 而小径级树木为了获取更多光 源, 可能将更多的氮储存在了植物叶片中, 用于提 高光合效率, 以促进横向生长。不过本实验并未讨 论不同径级叶片与土壤元素的关系, 关于小径级树 木是否将元素更多存储在叶片中仍需进一步研究。

\section{4 结论}

在研究限制元素对植物生长的影响时, 不同物 种以及不同径级树木对氮添加的响应不同。本研究 发现: 物种水平上, 氮添加促进了优势树种辽东栎 的生长; 在群落水平上, 树木生长对氮添加响应不 显著; 同时, 氮添加抑制了小径级 $(3-10 \mathrm{~cm})$ 树木的 生长。这一研究结果表明, 单纯从群落水平上研究 氮添加对树木生长的影响可能不够准确, 还需要充 分考虑不同物种和不同径级植物对氮的响应。由于 本实验并未对土壤微生物群落结构以及相关酶活性 开展相关研究。因此, 有关氮添加对北方温带森 林生态系统植物生长的影响机制还需进一步研究 讨论。

\section{参考文献}

Aber JD, Magill A, McNulty SG, Boone RD, Nadelhoffer KJ, Downs M, Hallett R (1995). Forest biogeochemistry and primary production altered by nitrogen saturation. Water, Air, \& Soil Pollution, 85, 1665-1670.

Aber JD, Nadelhoffer KJ, Steudler P, Melillo JM (1989). Nitrogen saturation in northern forest ecosystems. BioScience, 39, 378-386.

Alvarez-Clare S, Mack MC, Brooks M (2013). A direct test of nitrogen and phosphorus limitation to net primary productivity in a lowland tropical wet forest. Ecology, 94, 1540-1551.

BassiriRad H, Lussenhop JF, Sehtiya HL, Borden KK (2015). Nitrogen deposition potentially contributes to oak regeneration failure in the Midwestern temperate forests of the USA. Oecologia, 177, 53-63.

Bergkvist BO, Folkeson L (1992). Soil acidification and element fluxes of a Fagus sylvatica forest as influenced by simulated nitrogen deposition. Water, Air, \& Soil Pollution, 65, 111-133.

Burslem DFRP, Grubb PJ, Turner IM (1995). Responses to nutrient addition among shade-tolerant tree seedlings of lowland tropical rain forest in Singapore. Journal of Ecology, 83, 113-122.

Clark CM, Tilman D (2008). Loss of plant species after chronic low-level nitrogen deposition to prairie grasslands. Nature, 451, 712-715.

de Vries W, Du EZ, Butterbach-Bahl K (2014). Short and longterm impacts of nitrogen deposition on carbon sequestration by forest ecosystems. Current Opinion in Environmental Sustainability, 9-10, 90-104.

Deng MF, Liu LL, Sun ZZ, Piao SL, Ma YC, Chen YW, Wang J, Qiao CL, Wang X, Li P (2016). Increased phosphate uptake but not resorption alleviates phosphorus deficiency

www.plant-ecology.com 
induced by nitrogen deposition in temperate Larix principisrupprechtii plantations. New Phytologist, 212, 1019-1029.

Du EZ, Zhou Z, Li P, Hu XY, Ma YC, Wang W, Zheng CY, Zhu JX, He JS, Fang JY (2013). NEECF: A project of nutrient enrichment experiments in China's forests. Journal of Plant Ecology, 6, 428-435.

Du YG, Guo XW, Cao GM, Li YK (2016). Increased nitrous oxide emissions resulting from nitrogen addition and increased precipitation in an alpine meadow ecosystem. Polish Journal of Environmental Studies, 25, 447-451.

Gilliam FS (2006). Response of the herbaceous layer of forest ecosystems to excess nitrogen deposition. Journal of Ecology, 94, 1176-1191.

Gundersen P, Emmett BA, Kjønaas OJ, Koopmans CJ, Tietema A (1998). Impact of nitrogen deposition on nitrogen cycling in forests: A synthesis of NITREX data. Forest Ecology and Management, 101, 37-55.

Harpole WS, Ngai JT, Cleland EE, Seabloom EW, Borer ET, Bracken MES, Elser JJ, Gruner DS, Hillebrand H, Shurin JB, Smith JE (2011). Nutrient co-limitation of primary producer communities. Ecology Letters, 14, 852-862.

Högberg P, Fan HB, Quist M, Binkley D, Tamm CO (2006). Tree growth and soil acidification in response to 30 years of experimental nitrogen loading on boreal forest. Global Change Biology, 12, 489-499.

Jefts S, Fernandez IJ, Rustad LE, Dail DB (2004). Decadal responses in soil $\mathrm{N}$ dynamics at the Bear Brook Watershed in Maine, USA. Forest Ecology and Management, 189, 189-205.

Jung JY, Lal R, Jastrow JD, Tyler DD (2011). Nitrogenous fertilizer effects on soil structural properties under switchgrass. Agriculture Ecosystems \& Environment, 141, 215-220.

Kjønaas OJ, Stuanes AO, Huse M (1998). Effects of weekly nitrogen additions on $\mathrm{N}$ cycling in a coniferous forest catchment, Gårdsjön, Sweden. Forest Ecology and Management, 101, 227-249.

Koerselman W, Meuleman AFM (1996). The vegetation N:P ratio: A new tool to detect the nature of nutrient limitation. Journal of Applied Ecology, 33, 1441-1450.

Li Y, Tian DS, Yang H, Niu SL (2018). Size-dependent nutrient limitation of tree growth from subtropical to cold temperate forests. Functional Ecology, 32, 95-105.

Lu XK, Mo JM, Dong SF (2008). Effects of nitrogen deposition on forest biodiversity: A review. Acta Ecologica Sinica, 28, 5532-5548. [鲁显楷, 莫江明, 董少峰 (2008). 氮沉降对 森林生物多样性的影响. 生态学报, 28, 5532-5548.]

Magill AH, Aber JD, Currie WS, Nadelhoffer KJ, Martin ME, McDowell WH, Melillo JM, Steudler P (2004). Ecosystem response to 15 years of chronic nitrogen additions at the Harvard Forests LTER, Massachusetts, USA. Forest Ecology and Management, 196, 7-28.
Magnani F, Mencuccini M, Borghetti M, Berbigier P, Berninger F, Delzon S, Grelle A, Hari P, Jarvis PG, Kolari P, Kowalski AS, Lankreijer H, Law BE, Lindroth A, Loustau D, Manca G, Moncrieff JB, Rayment M, Tedeschi V, Valentini R, Grace J (2007). The human footprint in the carbon cycle of temperate and boreal forests. Nature, 447, 849-851.

Matson P, Lohse KA, Hall SJ (2002). The globalization of nitrogen deposition: Consequences for terrestrial ecosystems. AMBIO, 31, 113-119.

McConnaughay KDM, Coleman JS (1999). Biomass allocation in plants: Ontogeny or optimality? A test along three resource gradients. Ecology, 80, 2581-2593.

Nadelhoffer KJ, Emmett BA, Gundersen P, Kjønaas OJ, Koopmans CJ, Schleppi P, Tietema A, Wright RF (1999). Nitrogen deposition makes a minor contribution to carbon sequestration in temperate forests. Nature, 398, 145-148.

Reidsma P, Tekelenburg T, van den Berg M, Alkemade R (2006). Impacts of land-use change on biodiversity: An assessment of agricultural biodiversity in the European Union. Agriculture, Ecosystems \& Environment, 114, 86-102.

Solberg S, Dobbertin M, Reinds GJ, Lange H, Andreassen K, Fernandez PG, Hildingsson A, de Vries W (2009). Analyses of the impact of changes in atmospheric deposition and climate on forest growth in European monitoring plots: A stand growth approach. Forest Ecology and Management, 258, 1735-1750.

Stevens CJ (2004). Impact of nitrogen deposition on the species richness of grasslands. Science, 303, 1876-1879.

Su HX, Li GQ (2012). Simulating the response of the Quercus mongolica forest ecosystem carbon budget to asymmetric warming. Chinese Science Bulletin, 57, 1544-1552. [苏宏 新, 李广起 (2012). 模拟蒙古柇林生态系统碳收支对非 对称性升温的响应. 科学通报, 57, 1544-1552.]

Sun BH, Hu ZY, Lü JL, Zhou LN, Xu CK (2006). The leaching solution chemistry of a broad-leaved forest red soil under simulated $\mathrm{N}$ deposition in southern China. Acta Ecologica Sinica, 26, 1872-1881. [孙本华, 胡正义, 吕家珑, 周丽 娜, 徐成凯 (2006). 大气氮沉降对阔叶林红壤淋溶水化 学模拟研究. 生态学报, 26, 1872-1881.]

Tian D, Li P, Fang WJ, Xu J, Luo YK, Yan ZB, Zhu B, Wang JJ, Xu XN, Fang JY (2017). Growth responses of trees and understory plants to nitrogen fertilization in a subtropical forest in China. Biogeosciences, 14, 3461-3469.

Uriarte M, Canham CD, Thompson J, Zimmerman JK, Brokaw $\mathrm{N}$ (2005). Seedling recruitment in a hurricane-driven tropical forest: Light limitation, density-dependence and the spatial distribution of parent trees. Journal of Ecology, 93, 291-304.

Vitousek PM, Howarth RW (1991). Nitrogen limitation on land and in the sea: How can it occur? Biogeochemistry, 13, 
$87-115$.

Vitousek PM, Porder S, Houlton BZ, Chadwick OA (2010). Terrestrial phosphorus limitation: Mechanisms, implications, and nitrogen-phosphorus interactions. Ecological Applications, 20, 5-15.

Wang QSY, Zheng CY, Zhang XY, Zeng FX, Xing J (2016). Impacts of nitrogen addition on foliar nitrogen and phosphorus stoichiometry in a subtropical evergreen broadleaved forest in Mount Wuyi. Chinese Journal of Plant Ecology, 40, 1124-1135. [王乔姝怡, 郑成洋, 张歆阳, 曾发旭, 邢娟 (2016). 氮添加对武夷山亚热带常绿阔叶 林植物叶片氮磷化学计量特征的影响. 植物生态学报, 40, 1124-1135.]

Wang W, Li QK, Ma KP (2000). Establishment and spatial distribution of Quercus liaotungensis Koidz. seedlings in
Dongling Mountain. Acta Phytoecologica Sinica, 24, 595-600. [王巍, 李庆康, 马克平 (2000). 东灵山地区 辽东栋幼苗的建立和空间分布. 植物生态学报, 24 , 595-600.]

Wright RF, Rasmussen L (1998). Introduction to the NITREX and EXMAN projects. Forest Ecology and Management, $101,1-7$.

Wright SJ, Yavitt JB, Wurzburger N, Turner BL, Tanner EVJ, Sayer EJ, Santiago LS, Kaspari M, Hedin LO, Harms KE, Garcia MN, Corre MD (2011). Potassium, phosphorus, or nitrogen limit root allocation, tree growth, or litter production in a lowland tropical forest. Ecology, 92, 1616-1625.

责任编委: 唐志尧 责任编辑: 李 敏 实习编辑: 赵 航 